\title{
Establishment of embryonic shoot-root axis is involved in auxin and cytokinin response during Arabidopsis somatic embryogenesis
}

\author{
Ying Hua Su ${ }^{\ddagger}$, Yu Bo Liu ${ }^{\ddagger}$, Bo Bai ${ }^{\dagger}$ and Xian Sheng Zhang* \\ State Key Laboratory of Crop Biology, College of Life Sciences, Shandong Agricultural University, Taian, China
}

\author{
Edited by: \\ Kang Chong, Institute of Botany, The \\ Chinese Academy of Sciences, China

\section{Reviewed by:} \\ Venugopala Reddy Gonehal, \\ University of California, Riverside, \\ USA \\ Marcelo Carnier Dornelas, \\ Universidade Estadual de Campinas, \\ Brazil

\section{*Correspondence:} \\ Xian Sheng Zhang, State Key \\ Laboratory of Crop Biology, College of \\ Life Sciences, Shandong Agricultural \\ University, Taian, Shandong 271018, \\ China \\ e-mail: zhangxs@sdau.edu.cn \\ ${ }^{\dagger}$ Present address: \\ Bo Bai, Shandong Rice Research \\ Institute, Shandong Academy of \\ Agricultural Science, Jinan, Shandong, \\ China \\ FThese authors have contributed \\ equally to this work.
}

Auxin and cytokinin signaling participates in regulating a large spectrum of developmental and physiological processes in plants. The shoots and roots of plants have specific and sometimes even contrary responses to these hormones. Recent studies have clearly shown that establishing the spatiotemporal distribution of auxin and cytokinin response signals is central for the control of shoot apical meristem (SAM) induction in cultured tissues. However, little is known about the role of these hormones in root apical meristem (RAM) initiation. Here, we found that the expression patterns of several regulatory genes critical for RAM formation were correlated with the establishment of the embryonic root meristem during somatic embryogenesis in Arabidopsis. Interestingly, the early expression of the WUS-RELATED HOMEOBOX 5 (WOX5) and WUSCHEL genes was induced and was nearly overlapped within the embryonic callus when somatic embryos (SEs) could not be identified morphologically. Their correct expression was essential for RAM and SAM initiation and embryonic shoot-root axis establishment. Furthermore, we analyzed the auxin and cytokinin response during SE initiation. Notably, cytokinin response signals were detected in specific regions that were correlated with induced WOX5 expression and subsequent SE formation. Overexpression of the ARABIDOPSIS RESPONSE REGULATOR genes ARR7 and ARR15 (feedback repressors of cytokinin signaling), disturbed RAM initiation and SE induction. These results provide new information on auxin and cytokinin-regulated apical-basal polarity formation of shoot-root axis during somatic embryogenesis.

Keywords: shoot-root axis, root apical meristem, cytokinin response, auxin response, somatic embryogenesis, Arabidopsis

\section{INTRODUCTION}

The most critical event during embryogenesis appears to be the formation of the shoot apical meristem (SAM) and root apical meristem (RAM), from which almost the entire plant is postembryonically established (Meinke, 1991; Scheres, 2007). In the SAM of Arabidopsis, WUSCHEL (WUS) is a critical regulator, and encodes a homeodomain protein that is required for stem cell formation and maintenance (Laux et al., 1996). WUS is switched on in the four inner cells of the pro-embryo at the 16-cell globular stage, and is an early molecular marker for SAM initiation in the embryo (Weigel and Jürgens, 2002).

Root growth and development are sustained by the RAM, which is formed during embryogenesis (Sabatini etal., 2003; Petricka et al., 2012). Embryonic RAM formation is initiated at the globular stage, when the uppermost cell of the suspensor-the hypophysis - is recruited in the embryo proper. After asymmetrical division of the hypophysis, the small descendant cell gives rise to the quiescent center $(\mathrm{QC})$, which maintains stem cell identity in the surrounding cells of the RAM to produce a set of differentiated tissues (Möller and Weijers, 2009; Peris et al., 2010). Mutants that fail to form the hypophysis often produce rootless seedlings (Möller and Weijers, 2009). An element required in the QC to maintain columella stem cells is WUS-RELATED HOMEOBOX 5 (WOX5), a putative homeodomain transcription factor (Haecker et al., 2004). In the QC, WOX5 acts in a similar way to WUS in the organizing center (OC) of the SAM, highlighting molecular and developmental similarities between the stem cell niches of both root and shoot meristems (Perilli et al., 2012). In addition, several other putative transcription factors have been shown to contribute to embryonic RAM formation. PLETHORA (PLT) genes, which belong to the AP2-type transcription factor family, play a key role in the specification and maintenance of root stem cells from early embryogenesis onward (Aida et al., 2004; Galinha et al., 2007). Ectopic PLT expression in the embryo induces transformation of apical domain cells into root stem cells (Aida etal., 2004). The SCARECROW (SCR)/SHORTROOT (SHR) transcription factors are required to maintain stem cell activity within the RAM (Di Laurenzio etal., 1996; Helariutta et al., 2000; Sabatini et al., 2003).

Auxin and cytokinin are required for cell differentiation and specification during embryogenesis (Müller and Sheen, 2008; Möller and Weijers, 2009). Asymmetric distribution of auxin mediated by auxin polar transport establishes the apical-basal axis of the embryo, showing that auxin is required for pattern 
formation of the embryo (Friml et al., 2003; Möller and Weijers, 2009). Cytokinin signaling components function in the hypophysis at the early globular stage of the embryo (Müller and Sheen, 2008). After the first division of the hypophysis, the apical daughter cell maintains the phosphorelay activity of cytokinin signaling, whereas cytokinin signaling is repressed in the basal daughter cell. In early embryogenesis, auxin antagonizes cytokinin signaling through direct transcriptional activation of ARABIDOPSIS RESPONSE REGULATOR (ARR)7 and ARR15, feedback repressors of cytokinin signaling in the basal cell (Hwang and Sheen, 2001; Müller and Sheen, 2008; Buechel et al., 2010).

Somatic embryogenesis is generally believed to be mediated by a signaling cascade triggered by exogenous auxin (Skoog and Miller, 1957; Sugiyama, 1999, 2000). Indeed, our previous work has shown that the establishment of auxin gradients is correlated with induced WUS expression and subsequent embryonic SAM formation during somatic embryogenesis (Su et al., 2009). It has also been suggested that the establishment of the RAM in somatic embryos requires an appropriate auxin gradient (Bassuner et al., 2007). However, the mechanism by which root stem cell specification occurs during early somatic embryogenesis is far from understood. Here, we analyzed the expression patterns of a few critical marker genes involved in RAM formation and development during somatic embryogenesis. Besides the auxin gradients that are established in specific regions of embryonic callus, we found that the spatiotemporal cytokinin response was correlated with RAM formation. Such cytokinin response patterns were critical for spatial induction of RAM-specific genes, such as WOX5 and PLT, and subsequent RAM establishment in the embryonic callus. Our results reveal the distinct functions of cytokinin and auxin signaling required for RAM and SAM induction and shoot-root axis establishment during early somatic embryogenesis.

\section{MATERIALS AND METHODS PLANT MATERIALS}

All Arabidopsis mutants and transgenic lines used in this study were Columbia ecotypes. The $p$ WOX5::GFP, pPLT2::RFP, pSCR::GFP reporter lines and plt2-1 mutants were kindly provided by Dr. C. Li (Institute of Genetics and Developmental Biology, Chinese Academy of Sciences). pWUS::DsRED-N7 and DR5rev:3XVENUSN7 seeds were kindly provided by Dr E. M. Meyerowitz (Division of Biology, California Institute of Technology, Pasadena, CA, USA). The ahk2 ahk4 and ahk3 ahk4 mutants were obtained from Dr. C. Ueguchi (Bioscience and Biotechnology Center, Nagoya University, Nagoya, Japan). The DR5rev::GFP lines were provided by Dr J. Friml (Zentrum für Molekularbiologie der Pflanzen, Universität Tübingen, Germany). $p A R R 7:: G F P$ and pARR15::GFP seeds were provided by Dr J. Sheen (Harvard Medical School, USA). Double reporter lines were generated as follows: $p$ WOX5::GFP lines were crossed with $p W U S:: D s R E D-N 7$ lines; DR5rev:3XVENUS-N7 lines were crossed with $p$ WOX5::GFP lines, and $p P L T 2:: R F P$ lines were crossed with DR5rev::GFP lines; $p A R R 7:: G F P$ lines were crossed with $p W U S:: D s R E D-N 7$ lines.

\section{ANTISENSE WOX5 cDNA PLASMID CONSTRUCTION}

To determine the role of WOX5 during somatic embryo induction, a 754 bp cDNA fragment of the WOX5 coding region was amplified using the primers $5^{\prime}$-ATACTAGTAAACAGTTGAGGACTTTACA TC-3' (forward) and 5'-ATCTCGAGTACGCATTCCATAACATAG ATT-3' (reverse). The WOX5 antisense cDNA was then cloned into the estradiol inducible XVE binary vector (Zuo et al., 2000), and transformed into Arabidopsis plants.

\section{GROWTH CONDITIONS, SE INDUCTION AND CHEMICAL TREATMENTS}

The growth conditions for Arabidopsis plants and somatic embryo (SE) induction followed Su et al. (2009). To induce the transcription of inserted WOX5 antisense cDNAs, the primary somatic embryos (PSEs) were cultured in embryonic callus-inducing medium (ECIM) with $10 \mu \mathrm{M}$ estradiol (prepared in DMSO as $10 \mathrm{mM}$ stock; Sigma) for 14 days. Then, the cultured tissues were transferred to somatic embryo-inducing medium (SEIM) with $10 \mu \mathrm{M}$ estradiol for another 8 days. Estradiol was added every 2 days. The embryonic calli in SEIM were collected for phenotype observation.

\section{IN SITU HYBRIDIZATION}

Embryonic calli were fixed in FAA (10\% formaldehyde: 5\% acetic acid: $50 \%$ alcohol) overnight at $4^{\circ} \mathrm{C}$. After dehydration, the fixed callus tissues were embedded in paraffin (Sigma) and sectioned at $8 \mu \mathrm{m}$. Antisense and sense WOX5 probes were used for hybridization as previously described by Zhao et al. (2006). The primers used to amplify the 440-bp WOX5 probes were $5^{\prime}$-CATCATCATCAACCATCAACT- $3^{\prime}$ (forward) and $5^{\prime}$-CCATAACATAGATTCTTATATC-3' (reverse).

\section{IMAGING CONDITIONS}

Somatic embryo morphology was photographed using an Olympus JM dissecting microscope. To detect the fluorescence signals of the marker lines, a Zeiss 510 Meta laser scanning confocal microscope with a $20 \times$ air objective and a $40 \times$ oil-immersion lens was used. Specific sets of filters were selected as described previously by Heisler et al. (2005). The Zeiss LSM software was used to analyze the confocal images. At least 80 samples of each marker line were imaged to confirm the expression patterns at each stage.

\section{RESULTS}

\section{GENES FOR RAM SPECIFICATION ARE INDUCED DURING EARLY SOMATIC EMBRYOGENESIS}

Previously, we described a highly reproducible somatic embryogenesis system in Arabidopsis in detail (Su et al., 2009). Green PSEs can be generated from explants (immature zygotic embryos), and then disk-like embryonic calli are produced from PSEs in ECIM containing 2,4-dichlorophenoxyacetic acid (2,4-D). After the calli are transferred to 2,4-D-free SEIM, secondary somatic embryos (SSEs) are induced.

To analyze the spatiotemporally regulated formation of the root stem cell niche at the early stages of somatic embryogenesis, we examined the expression patterns of genes that play critical roles in root stem cell specification. Weak $p$ WOX5::GFP signals were detected in a few internal regions but not in the edge regions of embryonic callus grown in ECIM for 14 days (data not shown). In contrast, after the embryonic calli were transferred to SEIM, stronger GFP signals started to be detected in some small edge regions at around $24 \mathrm{~h}$ (Figure 1A). At this time, the pro-embryos 

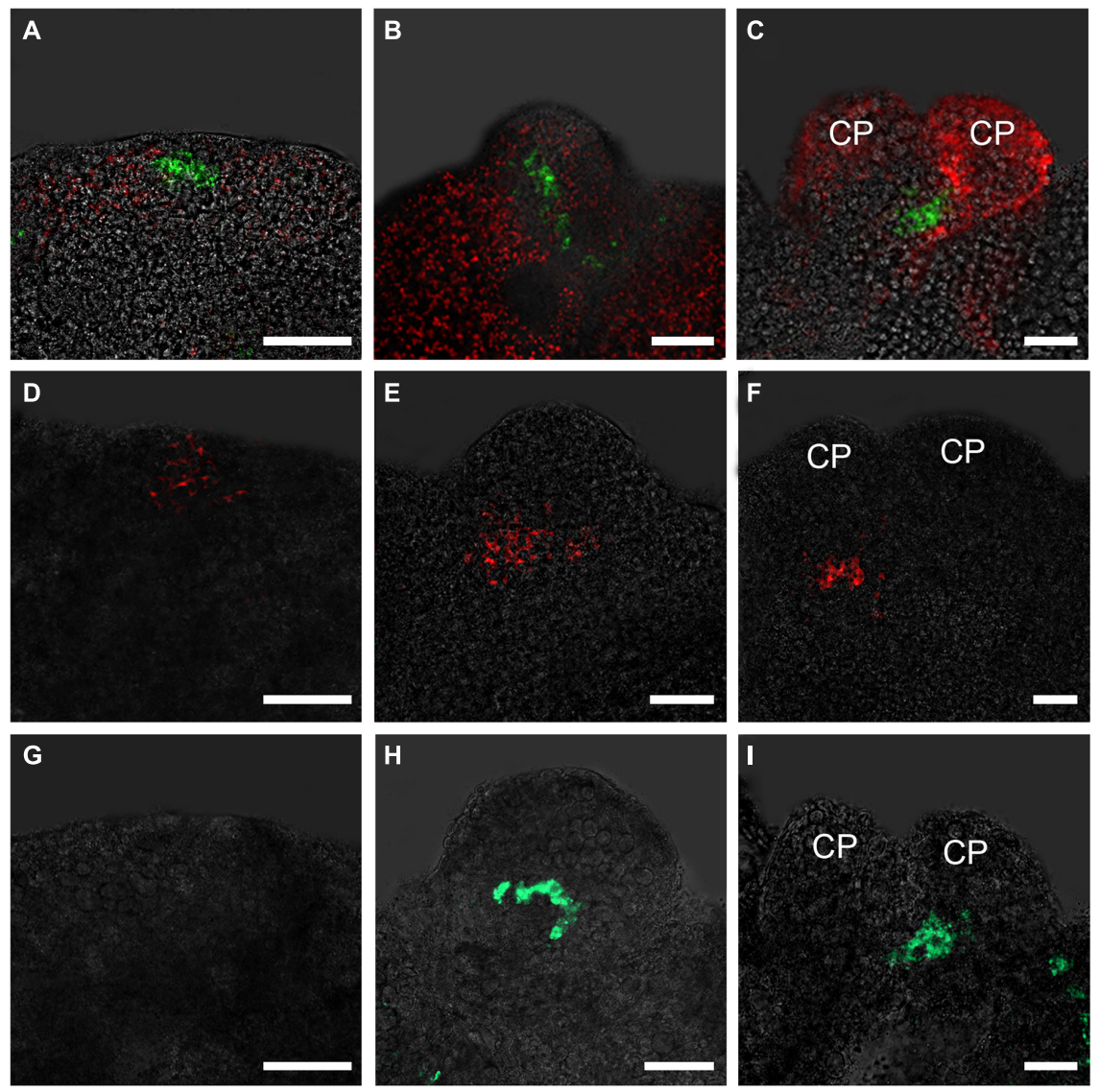

FIGURE 1 | Expression patterns of WOX5, PLT2, and SCR genes in embryonic calli during somatic embryogenesis. (A-C) Expression patterns of WOX5 indicated by pWOX5::GFP in embryonic calli induced in SEIM for $24 \mathrm{~h}$ (A; 83.72\%, $n=86$ ), 2 days (B; 84.27\%, $n=89$ ) and 3 days (C; $80.21 \%, n=96)$. (D-F) Expression patterns of PLT2 indicated by pPLT2::RFP in embryonic calli induced in somatic embryo-inducing medium
(SEIM) for $24 \mathrm{~h}$ (D; 87.65\%, $n=81), 2$ days (E; 84.95\%, $n=93$ ) and 3 days $(\mathbf{F} ; 90.53 \%, n=95)$. (G-I) Expression patterns of SCR indicated by pSCR::GFP in embryonic calli induced in SEIM for $24 \mathrm{~h}(\mathbf{G} ; 87.36 \%$, $n=87), 2$ days $(\mathbf{H} ; 89.58 \%, n=96)$ and 3 days $(\mathbf{I} ; 86.90 \%, n=84) . C P$, cotyledon primordia. Red signals in $\mathbf{A}-\mathbf{C}$ represent chlorophyll autofluorescence. Scale bars $=80 \mu \mathrm{m}$. were not identifiable morphologically, but the callus cells with WOX5 activity might have been the initial QC. Later, GFP signals were observed in the basal regions of the globular pro-embryos and then pro-embryos with cotyledon primordia (CP; Figures 1B,C). PLT2 exhibited similar spatial expression patterns to WOX5 at these stages (Figures 1D-F). Different from WOX5 expression, PLT2 expression was observed in a relatively large group of cells within the callus, which represented the root stem cell niche of the SE. We also determined the expression patterns of SCR, whose expression defines the position of the QC (Figures 1G-I). The GFP signals of SCR were first detected at 36-48 h after induction in SEIM, which was later than WOX5 and PLT2 expression. The expression patterns of genes for the QC and stem cell formation indicated that the RAM is established during SE induction.

\section{THE EMBRYONIC SHOOT-ROOT AXIS OF THE SE IS ESTABLISHED AT EARLY SOMATIC EMBRYOGENESIS}

To determine the relative expression domains of WUS and WOX5, we analyzed their co-localization using a $p W U S:: D s R E D-$ N7 pWOX5::GFP marker line. WUS and WOX5 transcription signals were first detected nearly overlapped at the edge regions of callus grown in SEIM for around $24 \mathrm{~h}$ (Figure 2A). At $36 \mathrm{~h}$, the WOX5 expression domain was just below and adjacent to that of WUS (Figure 2B). Subsequently, WUS transcripts were localized at the top regions between the $\mathrm{CP}$ of the pro-embryo, whereas WOX5 transcripts were localized at the basal regions (Figures 2C,D). Thus, the SAM and RAM were initiated early and nearly overlapped in the edge regions of the callus, indicating that the apical-basal polarity of SE is determined and an embryonic shoot-root axis is established at the early stages of somatic embryogenesis.

\section{RAM-SPECIFIC WOX5 AND PLT EXPRESSION IS REQUIRED FOR EMBRYONIC ROOT FORMATION AND SE INDUCTION}

To determine the roles of WOX5 during somatic embryogenesis, we constructed a vector carrying antisense WOX5 driven by an estradiol receptor-based transactivator, XVE (Zuo et al., 2000), and transferred it into plants. To monitor estradiol-induced production of cDNA-encoded transcripts, quantitative real-time PCR (qRT-PCR) was performed to detect expression levels of WOX5 

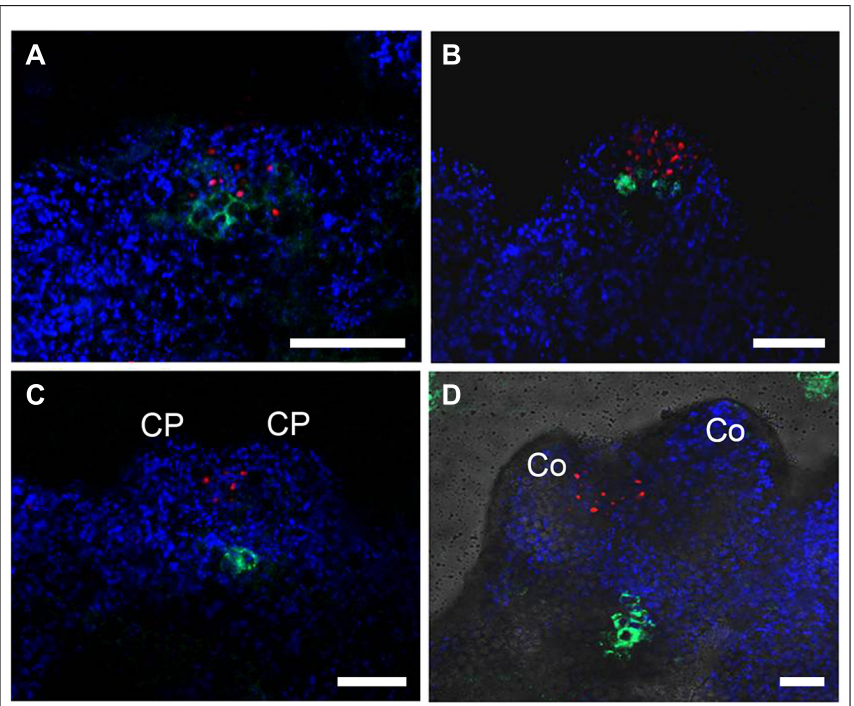

FIGURE 2 | Relative expression domains of WOX5 and WUS genes. pWOX5::GFP (green) and pWUS::DsRed-N7 (red) signals in embryonic calli induced in SEIM for $24 \mathrm{~h}(\mathbf{A} ; 86.81 \%, n=91), 2$ days (B; 85.39\%, $n=89)$, 3 days $(\mathbf{C} ; 89.66 \%, n=87)$ and 4 days (D; 87.37\%, $n=95)$; CP, cotyledon primordia; $\mathrm{Co}$, cotyledons. Blue signals represent chlorophyll autofluorescence. Scale bars $=80 \mu \mathrm{m}$.

in 15 days shoots of wild type (WT) and WOX5 antisense plants (Figure 3G). Estradiol was added every 2 days in medium. Immature zygotic embryos of the transgenic plants were used as explants. Green PSEs were induced on the shoot meristems of $84.47 \%$ of the explants after 10 days of culture on B5 agar medium containing $4.5 \mu \mathrm{M} 2,4-\mathrm{D}$ in light, without estradiol in the medium (Figure 3A; Table 1). After the PSEs were transferred from ECIM to SEIM, $62.26 \%$ of untreated calli produced SSEs and each embryonic callus generated 52.6 \pm 7.6 normal SSEs (Figure 3B; Table 1). However, most explants carrying the antisense WOX5 construct produced abnormal PSEs with deficient hypocotyl elongation and embryonic root formation in the presence of estradiol (Figure 3C; Table 1). Only $8.61 \%$ of embryonic calli carrying the antisense WOX5 construct produced SSEs, and each embryonic callus generated only $3.3 \pm 2.2$ normal SSEs (Figure 3D; Table 1). We also examined PLT2's function during SE induction. The plt2-1 mutants generated PSEs with abnormal hypocotyls and embryonic roots (Figure 3E), as described for plt1-1 plt2-1 double mutants (Su and Zhang, 2014). Embryonic calli of plt2-1 mutants produced severely abnormal SSEs, without cotyledons or SAMs (Figure 3F; Table 1). Likewise, no hypocotyl- or root-like structures were observed on these SSEs. These results suggested that both WOX5 and PLT2 are required for SE formation.

\section{SPATIOTEMPORAL DISTRIBUTION OF AUXIN RESPONSES IN EARLY SE INDUCTION}

Previously, we reported that auxin response gradients were established in specific regions of the embryonic callus, and were responsible for SE formation (Su etal., 2009). Furthermore, we showed that the spatiotemporal distribution of the auxin response was correlated with the induced WUS expression at
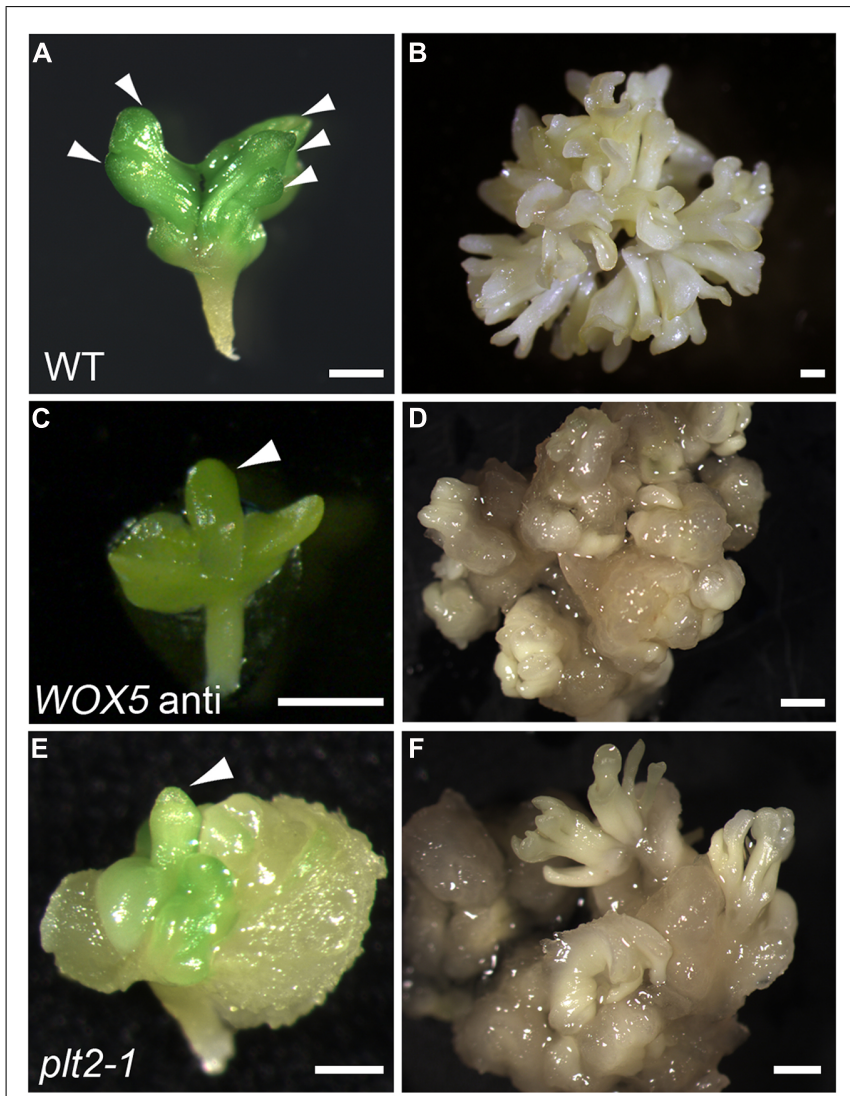

$\mathbf{G}$

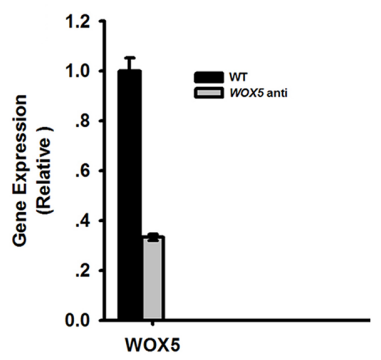

FIGURE 3 | Functional analysis of both WOX5 and PLT2 during somatic embryogenesis. (A,C,E) Phenotypes of primary somatic embryos (PSE) induction from wild type (WT; A), WOX5 antisense (C) and plt2-1 mutant (E) explants. Arrowheads indicate the PSE. (B,D,F) Phenotypes of SSE induction from WT (B), WOX5 antisense (D) and p/t2-1 mutant (F) calli grown on SEIM for 8 days. (G) Expression levels of WOX5 in

estradiol-induced 15 days shoots of WT and WOX5 antisense plants. Scale bars $=0.5 \mathrm{~mm}(\mathbf{A}, \mathbf{C}, \mathbf{E})$ and $1.2 \mathrm{~mm}(\mathbf{B}, \mathbf{D}, \mathbf{F})$.

early somatic embryogenesis. Interestingly, we observed hardly any auxin response signals in the basal part of the somatic pro-embryo. We analyzed the auxin response signals and WOX5 expression within the callus by double labeling with DR5rev:3XVENUS$N 7$ and $p W O X 5:: G F P$. After $16 \mathrm{~h}$ incubation in SEIM, auxin response signals were detectable at the edge regions of the callus, but no WOX5 signal could be detected (Figure 4A). At $24 \mathrm{~h}$ after induction, WOX5 signals were detected in the region just beneath the outermost cell layers, where auxin response signals were identified (Figure 4B). After $48 \mathrm{~h}$ incubation, 
Table 1 | Somatic embryo (SE) regeneration frequencies of different mutants and transgenetic lines.

\begin{tabular}{llllllll}
\hline Mutant $^{\text {a }}$ & Wild type & WOX5 anti & plt2-1 & 35S::ARR7 & 35S::ARR15 & ahk2 ahk4 & ahk3 ahk4 \\
\hline Ratio $^{b}$ & $84.47 \%$ & $18.79 \%$ & $25.56 \%$ & $34.14 \%$ & $26.67 \%$ & $32.23 \%$ \\
Ratio $^{c}$ & $62.26 \%$ & $8.61 \%$ & $23.50 \%$ & $21.05 \%$ & $17.70 \%$ & $15.95 \%$ \\
Number $^{d}$ & $52.6 \pm 7.6$ & $3.3 \pm 2.2$ & $17.2 \pm 7.3$ & $15.3 \pm 5.6$ & $13.5 \pm 7.1$ & $15.5 \pm 7.6$ & $12.2 \pm 4.3$
\end{tabular}

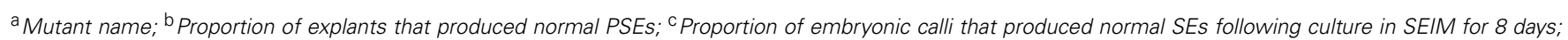

d Number of normal SEs produced per embryonic callus following culture in SEIM for 8 days (mean $\pm S D, n \geq 90$ ).
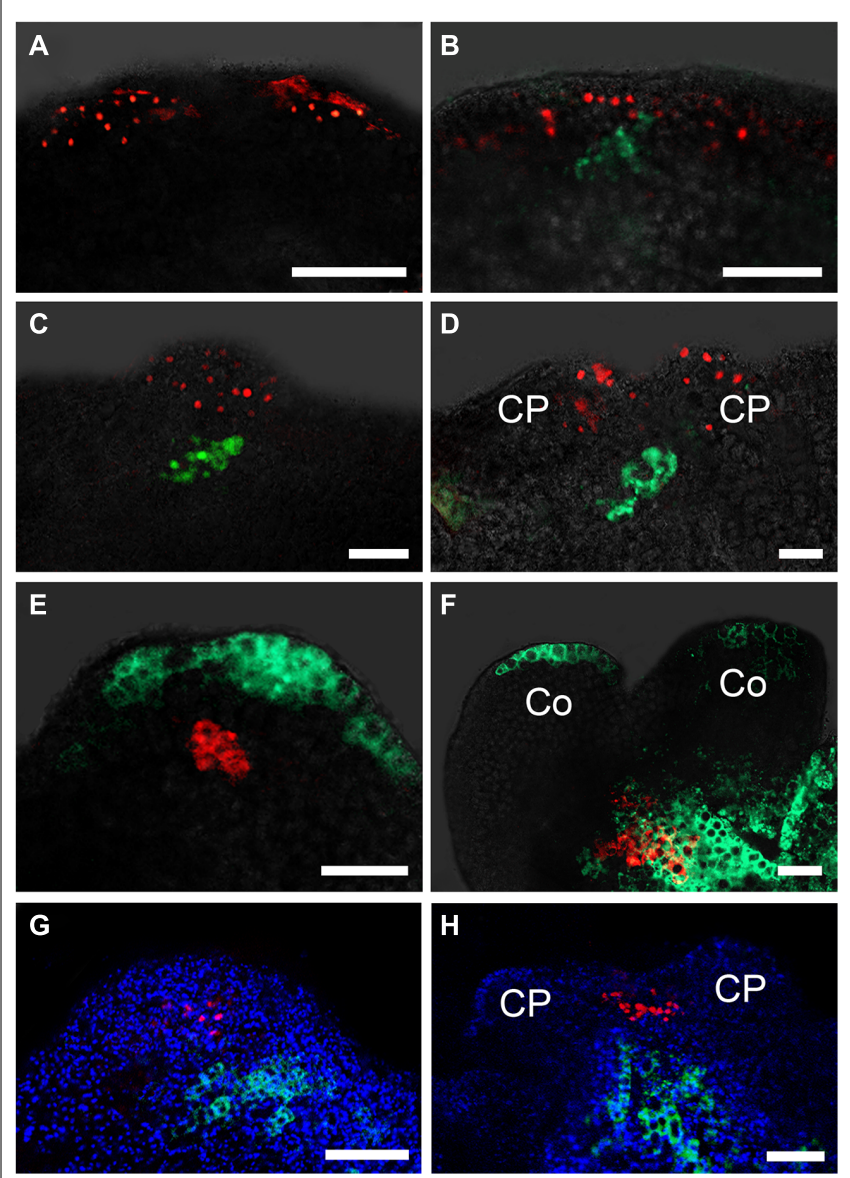

FIGURE 4 | Auxin and cytokinin responses in early somatic embryogenesis. (A-D) Auxin response represented by DR5rev:3XVENUS-N7 correlated with WOX5 induction represented by pWOX5::GFP in embryonic calli induced in SEIM for $16 \mathrm{~h}(\mathbf{A} ; 88.24 \%$, $n=85), 24 \mathrm{~h}(\mathbf{B} ; 83.33 \%, n=96), 2$ days (C; $87.50 \%, n=88)$ and 3 days (D; $89.66 \%, n=87$ ). pWOX5::GFP signals are in green,

DR5rev:3XVENUS-N7 fluorescence signals are in red. (E,F) Auxin response represented by DR5rev::GFP correlated with $P L T 2$ induction represented by $p P L T 2:: R F P$ in embryonic calli induced in SEIM for 3 days (E; $86.73 \%$, $n=98)$ and 4 days (F; $90.91 \%, n=77)$. DR5rev:: GFP fluorescence signals are in green, pPLT2::RFP signals are in red. $(\mathbf{G}, \mathbf{H})$ Cytokinin response represented by $p A R R 7:: G F P$ correlated with WUS induction represented by $p W U S:: D s R e d-N 7$ in embryonic calli induced in SEIM for 2 days (G; $90.43 \%, n=94)$ and 3 days $(\mathbf{H} ; 85.37 \%, n=82)$. pARR7::GFP

fluorescence signals are in green, pWUS::DsRed-N7 signals are in red, and chlorophyll autofluorescence is shown in blue. CP, cotyledon primordia; Co, cotyledons. Scale bars $=80 \mu \mathrm{m}$. strong auxin response signals were detected at the upper part of the pro-embryo, but WOX5 signals were localized at the basal part (Figure 4C). Later, auxin signals were redistributed to the top regions of the $\mathrm{CP}$, and WOX5 was continuously expressed in the basal part of the pro-embryo (Figure 4D). We also examined the auxin response in relation to PLT2 expression through double labeling with DR5rev:GFP and the PLT2 reporter $P P L T 2: R F P$. Until 4 days after induction in SEIM, there were auxin signals distributed at the basal region where PLT2 was expressed (Figures $4 \mathrm{E}, \mathbf{F}$ ). These results suggested that auxin response gradients were established in the SAM but not in the RAM of the pro-embryo during the early stages of somatic embryogenesis.

\section{CYTOKININ RESPONSES ARE SPATIOTEMPORALLY CORRELATED WITH RAM FORMATION}

It has been reported that both auxin and cytokinin responses are critical for specifying the root stem cell niche in embryos (Müller and Sheen, 2008). To determine how the cytokinin response occurs in callus when SEs are induced, we analyzed the spatiotemporal expression patterns of $A R R 7$ and $A R R 15$, which are primary responsive genes in cytokinin signaling and can be rapidly induced by cytokinin (To and Kieber, 2008; Werner and Schmülling, 2009). Signals of $p A R R 7:: G F P$ were first detected at some small regions of the calli near the edge at 24 and $36 \mathrm{~h}$ after induction in SEIM (Figures 5A,B), which was similar to the auxin response at these stages. Interestingly, after 2 days induction in SEIM, the signals were restricted to the basal part of the pro-embryo rather than the top (Figures 5C,D). We also used the pARR15::GFP reporter to examine the expression patterns of ARR15, and found similar distribution patterns of GFP signals to those of ARR7 (Figures 5E-H). These results showed that the cytokinin response occurs in the regions of SE initiation, but the cytokinin response patterns are different from those of the auxin response. To examine whether the cytokinin response is correlated with the establishment of the embryonic SAM, we visualized the cytokinin response using a $p A R R 7:: G F P$ pWUS:::DsRED-N7 marker line. The distribution regions of cytokinin signaling were quite different from those of WUS expression, which was localized in the opposite pole of the pro-embryos (Figures $\mathbf{4 G}, \mathbf{H}$ ). We further examined the cytokinin response in relation to WOX5 expression through double labeling with the PARR7::GFP and PWOX5::RFP reporters (Su and Zhang, 2014). Strong cytokinin responses were induced in the restrictive regions substantially overlapping with the WOX5 signals. Thus, the results suggest that establishment of the cytokinin response is 

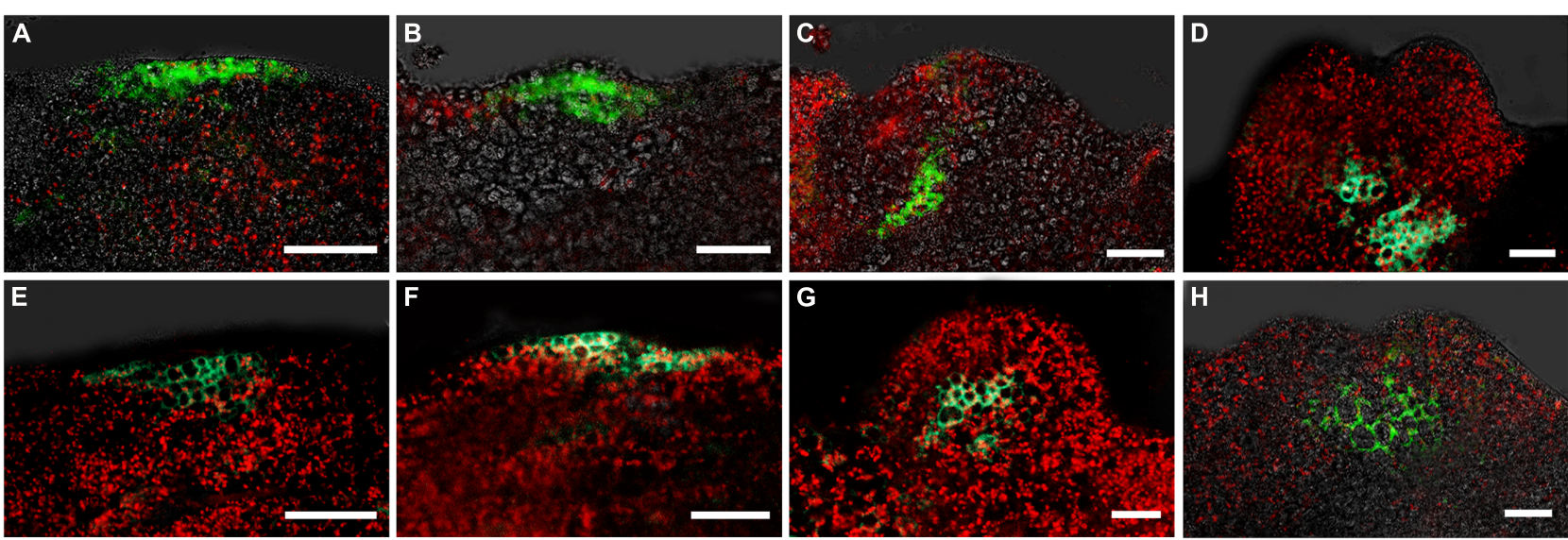

FIGURE 5 | Expression patterns of $A R R 7$ and $A R R 15$ during early somatic embryogenesis. (A-D) Expression patterns of $A R R 7$ indicated by pARR7::GFP in embryonic calli induced in SEIM for $16 \mathrm{~h} \mathrm{(A;93.02 \% ,} n=86)$, $24 \mathrm{~h}(\mathbf{B} ; 90.53 \%, n=95), 2$ days (C; 85.88\%, $n=85)$ and 3 days (D; 87.63\%,

correlated not with WUS but with WOX5 induction within the callus, implying that cytokinin is required for embryonic RAM initiation.

\section{CYTOKININ SIGNALING IS REQUIRED FOR EMBRYONIC RAM REGENERATION AND SE INDUCTION}

The spatial distribution of cytokinin responses detected through $A R R 7$ and ARR15 transcriptional signal profiles prompted us to confirm whether a functional cytokinin signaling mechanism is necessary for normal somatic embryogenesis. ARR7 and ARR15 act as negative regulators of cytokinin signaling by repressing type-B ARRs via unknown mechanisms (To and Kieber, 2008; Werner and Schmülling, 2009). Overexpression of ARR7 and $A R R 15$ can attenuate cytokinin signaling to a sufficiently low level, resulting in reduced sensitivity to cytokinin in root elongation and shoot formation or an early flowering phenotype (Werner and Schmülling, 2009). To facilitate functional analysis of cytokinin signaling, we generated transgenic plants overexpressing $A R R 7$ or ARR15 under the control of the CaMV 35S promoter. The ARR7overexpressing explants showed a severely defective PSE phenotype without normal elongated hypocotyls or obvious embryonic roots (Su and Zhang, 2014). Subsequently, 78.95\% of PSEs generated severely abnormal SSEs after induction in SEIM (Figure 6A; Table 1). Similar to ARR7-overexpressing plants, ARR15overexpressing plants also generated abnormal PSEs with defective hypocotyls and embryonic roots, and subsequently, abnormal SSEs (Su and Zhang, 2014; Figure 6B). In Arabidopsis, three histidine kinases (AHKs), AHK2, AHK3, and AHK4, positively regulate cytokinin-signaling as direct receptors of cytokinin (To and Kieber, 2008). Thus, we further analyzed the developmental characteristics of $a h k 2 a h k 4$ and $a h k 3 a h k 4$ double mutant calli during SE regeneration. The phenotypes of both double mutants were consistent with previous descriptions of $A R R 7$ - and $A R R 15$-overexpressing plants (Figures 6C,D; Table 1). Interestingly, we found that SE regeneration was impaired with defective cytokinin signaling, which was similar to WOX5-antisense plants and plt2-1 mutants.
Because the expression patterns of WOX5 were quite similar to the cytokinin response distribution, we hypothesized that cytokinin signaling might regulate WOX5 expression for early $\mathrm{SE}$ induction. We performed in situ hybridization to analyze WOX5 expression in the ahk3 ahk4 double mutant. Indeed, the WOX5 expression pattern was greatly disrupted in the ahk 3 ahk4 double mutant compared with the WT after the callus was transferred into SEIM (Figures 6E,F). WOX5 signals were restricted to the site of the future embryonic root meristem in the WT (Figure 6E), whereas the localization of WOX5 signals was stronger and more dispersed in the ahk3 ahk4 mutant (Figure 6F). These results indicated that cytokinin signaling negatively regulates WOX5 expression in the proper pattern for initiation of the embryonic RAM.

\section{DISCUSSION}

During somatic embryogenesis, the developmental process from the globular stage to the torpedo stage shares considerable similarity with that of zygotic embryogenesis (Meinke, 1991; Zimmerman, 1993). Although there are many similarities in the morphological and cellular programs of both zygotic and somatic embryogenesis, the mechanisms determining the initiation of these two processes might be different. The specific characteristics of somatic embryogenesis could be due to the origination of the somatic embryo from embryonic callus, not a zygote as in zygotic embryogenesis.

\section{THE ORIGINS OF EMBRYONIC SAM AND RAM WERE QUITE DIFFERENT BETWEEN SOMATIC EMBRYOS AND ZYGOTIC EMBRYOS}

In Arabidopsis, the mature embryo displays a main shoot-root axis of polarity, with the correct relative positioning of the embryonic SAM at the top and RAM at the opposite pole, separated by the hypocotyl (embryonic stem; Jürgens, 2001; Friml et al., 2003). The origin of this apical-basal pattern of shoot-root axis has been traced back to early embryogenesis, when zygotic division generates a smaller apical and a larger basal cell. After the apical domain 


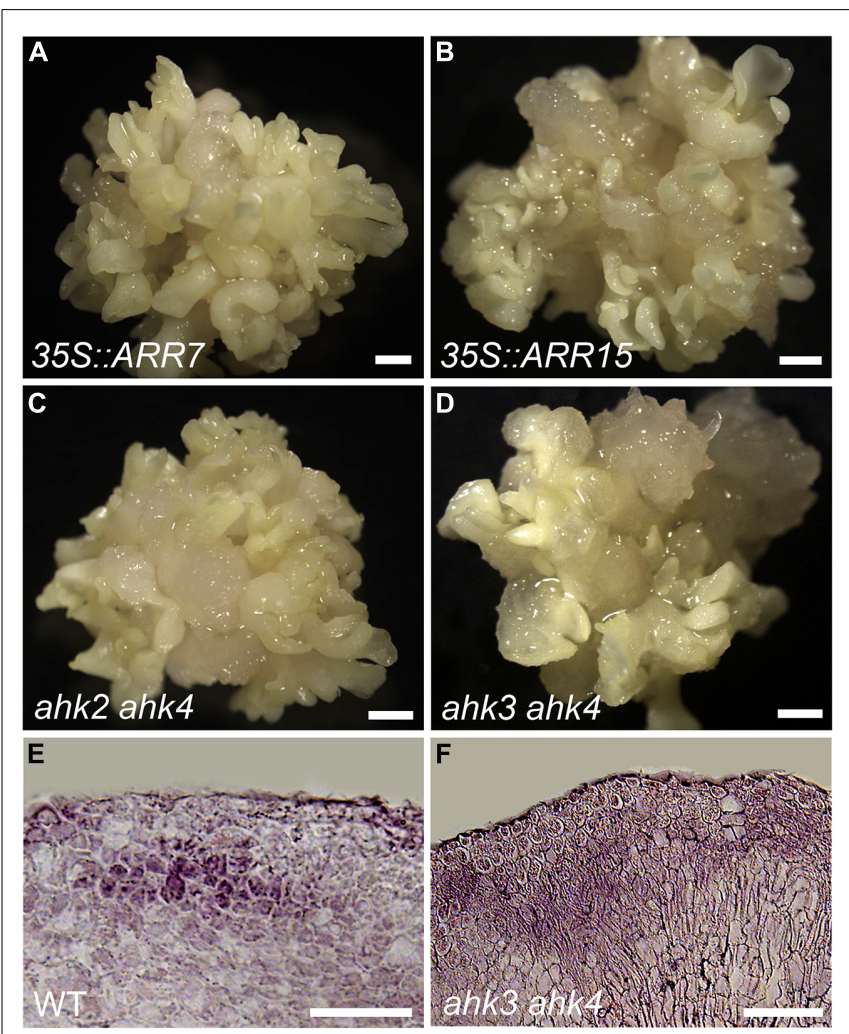

FIGURE 6 | Functions of cytokinin signaling on SE induction. (A-D) Phenotypes of SSE induction from 35S::ARR7 (A), 35S::ARR15 (B), ahk2 ahk4 mutant (C), and ahk3 ahk4 mutant (D) calli grown on SEIM for 8 days. (E) WOX5 transcript signals in WT embryonic callus cultured in SEIM for 24 h; 86.15\%, $n=35$. (F) Dispersed WOX5 transcript signals in ahk3 ahk4 double mutant callus following culture in SEIM for $24 \mathrm{~h} ; 87.30 \%, n=33$. Scale bars $=1.2 \mathrm{~mm}(\mathbf{A}-\mathbf{D})$ and $80 \mu \mathrm{m}(\mathbf{E}, \mathbf{F})$.

of the pro-embryo has been specified, the embryonic SAM is initiated by the onset of WUS expression in the four subepidermal apical cells of the 16-cell embryo (Mayer et al., 1998; Laux et al., 2004). Subsequently, the QC of the RAM is established at approximately late globular embryo stage and marked by the expression of WOX5 (Wysocka-Diller et al., 2000; Haecker et al., 2004).

During somatic embryogenesis, WUS and WOX5 were simultaneously activated in nearly overlapped callus cells, when somatic pro-embryos could not be identified morphologically (Figure 2; Su et al., 2009). The nearly overlapped spatial relationship between regenerated embryonic SAM and RAM during SE initiation represents the different origins of apical-basal pattern between somatic embryos and zygotic embryos. It is likely that SEs initiate from specific embryonic callus cells which acquire features similar to meristematic cells. These specific embryonic cells of callus are reprogrammed and determined to form cells of both OC and QC for embryonic SAM and RAM formation. In addition, early defects in RAM initiation with inhibited WOX5 expression also affected the initiation of the SAM, probably by disrupting the apical-basal pattern of early somatic embryogenesis. These results suggest that QC signaling not only maintains stem cell identity in the RAM but also is crucial for OC cells initiation, implying that the stem cell niches of the RAM and the SAM share developmental correlations during SE initiation.

\section{CYTOKININ RESPONSE WAS INVOLVED IN INDUCING CORRECT WOX5 EXPRESSION AND RAM FORMATION}

The patterns of embryonic SAM and RAM establishment in SE initiation suggest the presence of inductive hormonal signals to position them within the embryonic callus. Given the positive effects of auxin on WUS expression and SE induction (Su etal., 2009; Figure 4), it is likely a candidate factor that is required for embryonic RAM formation. Here, we found a cytokinin response distribution established in the regions where WOX5 and PLT2 were initiated (Figures 1 and 5). RAM formation and SE regeneration were severely inhibited in transgenic plants overexpressing $A R R 7$ or $A R R 15$ and in the ahk mutants, in which cytokinin signaling was inhibited (Figure 6). Moreover, in cultured tissues of the ahk mutants, WOX5 expression patterns were seriously disturbed compared with control tissues (Figure 6). Thus, we hypothesize that removal of exogenous auxin may be a stress factor that causes cytokinin polar distribution and responses in specific regions, which induce correct WOX5 expression and subsequent SE initiation. Induced WOX5 transcripts were continuously detectable in areas of high cytokinin response (Su and Zhang, 2014), suggesting that cytokinin functions in the initiation and maintenance of the embryonic RAM during somatic embryogenesis. The positive action of cytokinin in SAM regeneration has been reported in several studies (Pernisová et al., 2009; Buechel et al., 2010). Treatment with high levels of exogenous cytokinin induces cell proliferation and stimulates shoot regeneration (Skoog and Miller, 1957). Cytokinin induces WUS expression during in vitro establishment of the SAM from cultured root explants (Gordon et al., 2009). A cytokinin response occurs in the center of the regenerated SAM, overlapping with WUS expression regions (Cheng et al., 2013). In contrast, an opposite effect of cytokinin in root regeneration has been observed. Cytokinin influences auxin-induced RAM regeneration via regulation of PIN-mediated auxin polar transport (Pernisová et al., 2009). Therefore, the functions of cytokinin in RAM establishment during SE initiation differ from those in shoot and root regeneration.

\section{SPATIOTEMPORAL DISTRIBUTION OF AUXIN AND CYTOKININ RESPONSE IN EMBRYONIC CALLUS DETERMINES ESTABLISHMENT OF SE SHOOT-ROOT AXIS}

Cytokinin and auxin appear to be the most important hormones in the regulation of organ regeneration (Moubayidin et al., 2009; Su etal., 2011). A high exogenous auxin/cytokinin ratio induces root regeneration, whereas a low ratio promotes shoot induction (Skoog and Miller, 1957). Recent studies have suggested that exogenous hormones treatment is the critical factor triggering biosynthesis and response of endogenous hormones in early developmental events of in vitro regeneration. Specialized endogenous hormonal signaling is required for specific cell differentiation that determines the developmental fate of callus cells (Gordon etal., 2007; Su et al., 2009, 2011). During early somatic embryogenesis, removal of exogenous auxin triggers the regional distribution of endogenous auxin response 
in callus surrounding areas of WUS expression initiation ( $\mathrm{Su}$ et al., 2009). Following WUS induction, distribution of auxin response was re-established in the SAM region. In contrast, the distribution of cytokinin-response signal in callus overlapped with the areas of WOX5 expression ( $\mathrm{Su}$ and Zhang, 2014). These results imply that establishment of auxin and cytokinin response patterns within callus plays an important role in WUS and WOX5 regional expression and shoot-root axis formation. Furthermore, auxin response signals accumulated at the basal region of pro-embryos following prolonged incubation in SEIM (Figures 4E,F). The redistributed auxin response corresponded to PLT2 expression at the later stages of SE development. Therefore, our results suggest that cytokinin and auxin are key players in axial patterning of the SE, especially in shoot and root meristem initiation. The mechanisms of hormonal regulation in SE initiation are quite different from those in shoot or root regeneration individually, which remains a major challenge for the future.

\section{AUTHOR CONTRIBUTIONS}

Ying Hua Su and Xian Sheng Zhang designed the research. Ying Hua Su and Yu Bo Liu performed the research. Bo Bai analyzed the data. Ying Hua Su and Xian Sheng Zhang wrote the paper.

\section{ACKNOWLEDGMENT}

We are grateful to all members for their assistance in our laboratory. This research was supported by grants from the National Natural Science Foundation of China (90917015, 91217308, and 31170272).

\section{REFERENCES}

Aida, M., Beis, D., Heidstra, R., Willemsen, V., Blilou, I., Galinha, C., et al. (2004). The PLETHORA genes mediate patterning of the Arabidopsis root stem cell niche. Cell 119, 109-120. doi: 10.1016/j.cell.2004.09.018

Bassuner, B. M., Lam, R., Lukowitz, W., and Yeung, E. C. (2007). Auxin and root initiation in somatic embryos of Arabidopsis. Plant Cell Rep. 26, 1-11. doi: 10.1007/s00299-006-0207-5

Buechel, S., Leibfried, A., To, J. P., Zhao, Z., Andersen, S. U., Kieber, J. J., et al. (2010). Role of A-type ARABIDOPSIS RESPONSE REGULATORS in meristem maintenance and regeneration. Eur. J. Cell Biol. 89, 279-284. doi: 10.1016/j.ejcb.2009.11.016

Cheng, Z. J., Wang, L., Sun, W., Zhang, Y., Zhou, C., Su, Y. H., et al. (2013). Pattern of auxin and cytokinin responses for shoot meristem induction results from the regulation of cytokinin biosynthesis by AUXIN RESPONSE FACTOR3. Plant Physiol. 161, 240-251. doi: 10.1104/pp.112.203166

Di Laurenzio, L., Wysocka-Diller, J., Malamy, J. E., Pysh, L., Helariutta, Y., Freshour, G., et al. (1996). The SCARECROW gene regulates an asymmetric cell division that is essential for generating the radial organization of the Arabidopsis root. Cell 86, 423-433. doi: 10.1016/S0092-8674(00)80115-4

Friml, J., Vieten, A., Sauer, M., Weijers, D., Schwarz, H., Hamann, T., et al. (2003). Efflux-dependent auxin gradients establish the apical-basal axis of Arabidopsis. Nature 426, 147-153. doi: 10.1038/nature02085

Galinha, C., Hofhuis, H., Luijten, M., Willemsen, V., Blilou, I., Heidstra, R., et al. (2007). PLETHORA proteins as dose-dependent master regulators of Arabidopsis root development. Nature 449, 1053-1057. doi: 10.1038/nature06206

Gordon, S. P., Chickarmane, V. S., Ohno, C., and Meyerowitz, E. M. (2009). Multiple feedback loops through cytokinin signaling control stem cell number within the Arabidopsis shoot meristem. Proc. Natl. Acad. Sci. U.S.A. 106, 16529-16534. doi: 10.1073/pnas.0908122106

Gordon, S. P., Heisler, M. G., Reddy, G. V., Ohno, C., Das, P., and Meyerowitz, E. M. (2007). Pattern formation during de novo assembly of the Arabidopsis shoot meristem. Development 134, 3539-3548. doi: 10.1242/dev.010298
Haecker, A., Gross-Hardt, R., Geiges, B., Sarkar, A., Breuninger, H., Herrmann, M., et al. (2004). Expression dynamics of WOX genes mark cell fate decisions during early embryonic patterning in Arabidopsis thaliana. Development 131, 657-668. doi: 10.1242/dev.00963

Heisler, M. G., Ohno, C., Das, P., Sieber, P., Reddy, G. V., Long, J. A., et al. (2005). Patterns of auxin transport and gene expression during primordium development revealed by live imaging of the Arabidopsis inflorescence meristem. Curr. Biol. 15, 1899-1911. doi: 10.1016/j.cub.2005.09.052.

Helariutta, Y., Fukaki, H., Wysocka-Diller, J., Nakajima, K., Jung, J., Sena, G., et al. (2000). The SHORT-ROOT gene controls radial patterning of the Arabidopsis root through radial signaling. Cell 101, 555-567. doi: 10.1016/S0092-8674(00)80865$\mathrm{X}$

Hwang, I., and Sheen, J. (2001). Two-component circuitry in Arabidopsis cytokinin signal transduction. Nature 413, 383-389. doi: 10.1038/35096500

Jürgens, G. (2001). Apical-basal pattern formation in Arabidopsis embryogenesis. EMBO J. 20, 3609-3616. doi: 10.1093/emboj/20.14.3609

Laux, T., Mayer, K. F., Berger, J., and Jurgens, G. (1996). The WUSCHEL gene is required for shoot and floral meristem integrity in Arabidopsis. Development 122, 87-96.

Laux, T., Wurschum, T., and Breuninger, H. (2004). Genetic regulation of embryonic pattern formation. Plant Cell 16, S190-S202. doi: 10.1105/tpc. 016014

Mayer, K. F., Schoof, H., Haecker, A., Lenhard, M., Jurgens, G., and Laux, T. (1998). Role of WUSCHEL in regulating stem cell fate in the Arabidopsis shoot meristem. Cell 95, 805-815. doi: 10.1016/S0092-8674(00)81703-1

Meinke, D. W. (1991). Perspectives on genetic analysis of plant embryogenesis. Plant Cell 3, 857-866. doi: 10.1105/tpc.3.9.857

Möller, B., and Weijers, D. (2009). Auxin control of embryo patterning. Cold Spring Harb. Perspect. Biol. 1:a001545. doi: 10.1101/cshperspect. a001545

Moubayidin, L., Di Mambro, R., and Sabatini, S. (2009). Cytokinin-auxin crosstalk. Trends Plant Sci. 14, 557-562. doi: 10.1016/j.tplants.2009.06.010

Müller, B., and Sheen, J. (2008). Cytokinin and auxin interaction in root stemcell specification during early embryogenesis. Nature 453, 1094-1097. doi: 10.1038/nature06943

Perilli, S., Di Mambro, R., and Sabatini, S. (2012). Growth and development of the root apical meristem. Curr. Opin. Plant Biol. 15, 17-23. doi: 10.1016/j.pbi.2011.10.006

Peris, C. I., Rademacher, E. H., and Weijers, D. (2010). Green beginnings - pattern formation in the early plant embryo. Curr. Top. Dev. Biol. 91, 1-27. doi: 10.1016/S0070-2153(10)91001-6

Pernisová, M., Klima, P., Horak, J., Valkova, M., Malbeck, J., Soucek, P., et al. (2009). Cytokinins modulate auxin-induced organogenesis in plants via regulation of the auxin efflux. Proc. Natl. Acad. Sci. U.S.A. 106, 3609-3614. doi: 10.1073/pnas.0811539106

Petricka, J. J., Winter, C. M., and Benfey, P. N. (2012). Control of Arabidopsis root development. Annu. Rev. Plant Biol. 63, 563-590. doi: 10.1146/annurev-arplant042811-105501

Sabatini, S., Heidstra, R., Wildwater, M., and Scheres, B. (2003). SCARECROW is involved in positioning the stem cell niche in the Arabidopsis root meristem. Genes Dev. 17, 354-358. doi: 10.1101/gad.252503.

Scheres, B. (2007). Stem-cell niches: nursery rhymes across kingdoms. Nat. Rev. Mol. Cell Biol. 8, 345-354. doi: 10.1038/nrm2164

Skoog, F., and Miller, C. O. (1957). Chemical regulation of growth and organ formation in plant tissues cultured in vitro. Symp. Soc. Exp. Biol. 11, $118-130$.

Su, Y. H., Liu, Y. B., and Zhang, X. S. (2011). Auxin-cytokinin interaction regulates meristem development. Mol. Plant. 4, 616-625. doi: 10.1093/mp/ssr007

Su, Y. H., and Zhang, X. S. (2014). The hormonal control of regeneration in plants. Curr. Top. Dev. Biol. 108, 35-69. doi: 10.1016/B978-0-12-391498-9. 00010-3

Su, Y. H., Zhao, X. Y., Liu, Y. B., Zhang, C. L., O’neill, S. D., and Zhang, X. S. (2009). Auxin-induced WUS expression is essential for embryonic stem cell renewal during somatic embryogenesis in Arabidopsis. Plant J. 59, 448-460. doi: 10.1111/j.1365-313X.2009.03880.x

Sugiyama, M. (1999). Organogenesis in vitro. Curr. Opin. Plant Biol. 2, 61-64. doi: $10.1016 /$ S1369-5266(99)80012-0

Sugiyama, M. (2000). Genetic analysis of plant morphogenesis in vitro. Int. Rev. Cytol. 196, 67-84. doi: 10.1016/S0074-7696(00)96002-9 
To, J. P. C., and Kieber, J. J. (2008). Cytokinin signaling: two-components and more. Trends Plant Sci. 13, 85-92. doi: 10.1016/j.tplants.2007.11.005

Weigel, D., and Jürgens, G. (2002). Stem cells that make stems. Nature 415, 751-754. doi: $10.1038 / 415751$ a

Werner, T., and Schmülling, T. (2009). Cytokinin action in plant development. Curr. Opin. Plant Biol. 12, 527-538. doi: 10.1016/j.pbi.2009. 07.002

Wysocka-Diller, J. W., Helariutta, Y., Fukaki, H., Malamy, J. E., and Benfey, P. N. (2000). Molecular analysis of SCARECROW function reveals a radial patterning mechanism common to root and shoot. Development 127, 595-603.

Zhao, X. Y., Cheng, Z. J., and Zhang, X. S. (2006). Overexpression of TaMADS1, a SEPALLATA-like gene in wheat, causes early flowering and the abnormal development of floral organs in Arabidopsis. Planta 223, 698-707. doi: 10.1007/s00425-005-0123-x

Zimmerman, J. L. (1993). Somatic embryogenesis: a model for early development in higher plants. Plant Cell 5, 1411-1423. doi: 10.1105/tpc.5. 10.1411

Zuo, J., Niu, Q. W., and Chua, N. H. (2000). Technical advance: an estrogen receptor-based transactivator XVE mediates highly inducible gene expression in transgenic plants. Plant J. 24, 265-273. doi: 10.1046/j.1365-313x.2000. 00868.x

Conflict of Interest Statement: The authors declare that the research was conducted in the absence of any commercial or financial relationships that could be construed as a potential conflict of interest.

Received: 24 October 2014; accepted: 19 December 2014; published online: 14 January 2015.

Citation: Su YH, Liu YB, Bai B and Zhang XS (2015) Establishment of embryonic shoot-root axis is involved in auxin and cytokinin response during Arabidopsis somatic embryogenesis. Front. Plant Sci. 5:792. doi: 10.3389/fpls.2014.00792

This article was submitted to Plant Evolution and Development, a section of the journal Frontiers in Plant Science.

Copyright (C) 2015 Su, Liu, Bai and Zhang. This is an open-access article distributed under the terms of the Creative Commons Attribution License (CC BY). The use, distribution or reproduction in other forums is permitted, provided the original author(s) or licensor are credited and that the original publication in this journal is cited, in accordance with accepted academic practice. No use, distribution or reproduction is permitted which does not comply with these terms. 